\title{
Foetal Thigh Circumference: A Critical Evaluation of Its Relationship to Menstrual Age
}

\author{
Saryu Gupta ${ }^{1}$, Manoj Mathur ${ }^{2}$, Puneet Gambhir ${ }^{3}$, Bhardwaj $^{4}$ \\ ${ }^{1}$ Assistant Professor, Department of Radiodiagnosis and Imaging, Government Medical College and Rajindra \\ Hospital, Patiala (Punjab) India \\ ${ }^{2}$ Associate Professor, Department of Radiodiagnosis and Imaging, Government Medical College and Rajindra \\ Hospital, Patiala (Punjab) India \\ ${ }^{3}$ Assistant Professor, Department of Community Medicine, Government Medical College and Rajindra Hospital, \\ Patiala (Punjab) India \\ ${ }^{4}$ Ex-Professor and Head, Department of Radiodiagnosis and Imaging, Government Medical College and \\ Rajindra Hospital, Patiala (Punjab) India
}

\begin{abstract}
Accurate knowledge of foetal growth is clinically relevant to aid evaluation of diagnostic measurements of foetal growth and to identify potentially compromised gestations so as to enable timely intervention and management protocols. Intrauterine growth retardation is most commonly defined on the basis of a weight below the $10^{\text {th }}$ percentile for gestational age. Growth retarded foetuses are at increased risk for perinatal morbidity and mortality. The evaluation of individual thigh circumference measurements and identification of foetuses with reduced soft tissue mass require definition of a growth curve for this in normally growing foetuses which the present study intends to achieve.
\end{abstract}

Keywords: Ultrasound, foetal thigh circumference, IUGR

\section{Introduction}

Diagnostic ultrasound has a pivotal and ever expanding role as a decidual tool in modern day obstetrics, with its potential, both in the identification of foetal abnormalities as well as providing reassurance of foetal wellbeing. Given but one choice from the many biochemical and biophysical techniques that have been developed in more recent years towards improving pregnancy outcome, sonography would seem the best. Ultrasound is a non-ionizing, non-invasive, safe, rapid, accurate, convenient, reproducible and highly portable means of delineating foetal and placental details as well as prediction of gestational age which is of immense use both to the pregnant woman as well as to her obstetrician. So much so, that the scanning equipment has now become an extension of the obstetrician's eyes, ears and hands

Assessment of foetal growth requires the measurement of various parameters, each reflecting a different aspect of foetal development. One of these parameters is the thigh circumference, which reflects soft tissue mass as indicated by various pediatric studies. Since IUGR is primarily manifest as a loss of soft tissue mass in some patients, measurement of the foetal thigh circumference has the potential for identifying foetuses that might not be detected by other methods

\section{Material And Methods}

A prospective study was carried out on an ethnically, socio-economically and culturally mixed group of maternal study population of 287 subjects coming to the Department of Radiodiagnosis and Imaging via Gynaecology \& Obstetrics outdoors and wards of Rajendra Hospital, Patiala. Three main criteria were followed for inclusion into the study:

- Maternal menstrual history indicating normal last menstrual period and regular menses.

- Age of the foetus determined from menstrual history and biparietal diameter (BPD) measurement is in agreement within the range of expected variability.

- There is no evidence of abnormal growth based on

measurements of the head circumference (HC), abdominal circumference (AC) or femur length $\langle$ FL $\rangle$.

Foetuses were not subdivided by sex but only singleton pregnancies were considered. Subjects undergoing more than one ultrasound examination during the 22-40 weeks' gestational age range of this study were entered in the data base only once. Linear regression analysis was used to determine the optimal functions relating thigh circumference to menstrual age. The ThC measurements were made at the junction of the upper and middle thirds of the thigh where a change in femur profile shape could be detected; by direct measurement. 


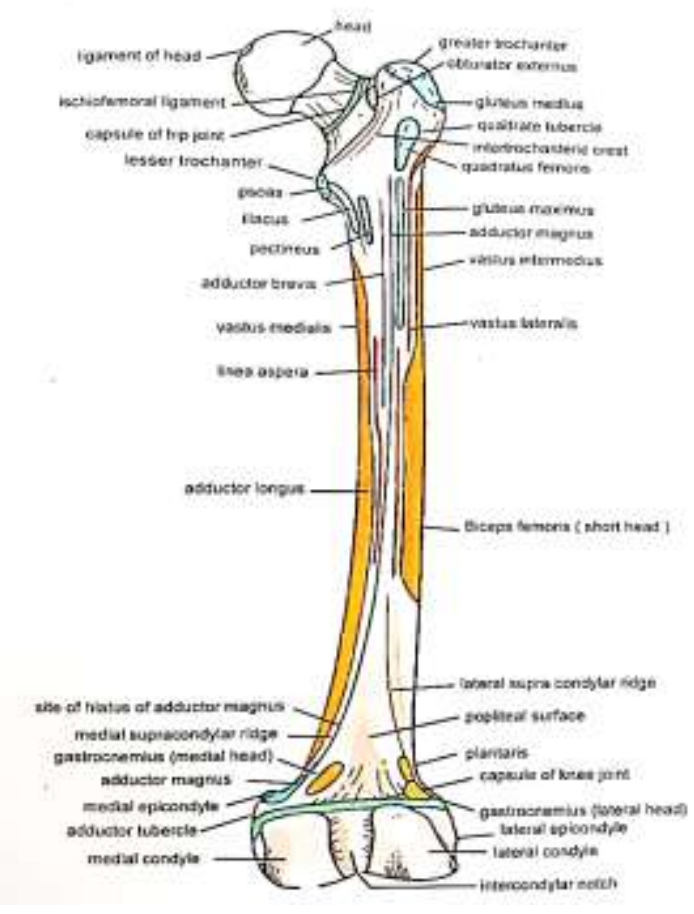

Figure 1: Transitional plane: anatomical dissections and cross sections indicated that this change was consistently observed distal to the cartilaginous part of the proximal end of the femur at the insertion of the Adductor Longus into the Linea Aspera approximately at the level of the proximal nutrient foramen. the change in bone profile (polygonal to oval / round) is likely due to muscle attachments in this area.

As most thigh profiles, did not have simple geometrical shapes, all thigh circumferences were determined by direct measurement. The $\mathrm{ThC}$ was measured twice by the same investigator in each image to rule out intra-observer variation. To assess the precision of the measurement process, the differences between pairs of measurements made on the same image were determined and expressed as a percentage of the first measurement. The mean and standard deviation of these percent deviations were calculated using standard statistical methods. Only data from one examination per foetus were included in the data set used to establish the ThC growth curve. Linear regression analysis was used to determine the optimal functions relating ThC to MA. Functions were evaluated based on their coefficient of determination (R), whether or not the coefficient values were statistically different $(\mathrm{p}<0.05)$ from zero as determined by ' $\mathrm{t}$ ' test, and the pattern of variability around the regression line, as indicated by deviation plots. Optimal models were those with the highest R2, all coefficients significantly different than zero, and deviation plots that showed uniform variability over the time interval studied.

\section{Observations}

The present study was carried out on 287 patients coming through the Department of Obstetrics and Gynaecology, Government Medical College/Rajendra Hospital, Patiala. Criteria for inclusion of patients were as given in material and methods.

On ultrasound, the following observations were made:

1. Determination of gestational age by measurement of

(a) Biparietal diameter

(b) Femur length

(c) Head circumference

(d) Abdominal circumference

2. Presentation of the foetus

3. Placental localization

4. Liquor amnii assessment

5. Craniovertebral junction (for any gross congenital anomaly)

6. Measurement of the foetal thigh circumference 
Evaluation of individual ThC measurements was done and a growth curve was defined for the same in normally growing foetuses, so as to enable identification of foetuses with reduced soft tissue mass.

\begin{tabular}{|c|c|c|}
\hline S.no & Menstrual age (weeks) & Number of patients \\
\hline 1 & 22 & 11 \\
\hline 2 & 23 & 20 \\
\hline 3 & 24 & 19 \\
\hline 4 & 25 & 16 \\
\hline 5 & 26 & 16 \\
\hline 6 & 27 & 15 \\
\hline 7 & 28 & 20 \\
\hline 8 & 29 & 21 \\
\hline 9 & 30 & 16 \\
\hline 10 & 31 & 15 \\
\hline 11 & 32 & 19 \\
\hline 12 & 33 & 14 \\
\hline 13 & 34 & 16 \\
\hline 14 & 35 & 21 \\
\hline 15 & 36 & 21 \\
\hline 16 & 37 & 14 \\
\hline 17 & 38 & 8 \\
\hline 18 & 39 & 4 \\
\hline 19 & 40 & 1 \\
\hline
\end{tabular}

Table 1: Menstrual age wise distribution of patients

\begin{tabular}{|c|c|c|c|}
\hline \multirow{2}{*}{$\begin{array}{l}\text { Menstrual age } \\
\text { (weeks) }\end{array}$} & \multicolumn{3}{|c|}{ Foetal Thigh Circumference } \\
\cline { 2 - 4 } & Lower limit & Predicted value & upper limit \\
\hline 22 & 5.9 & 7.1 & 8.4 \\
\hline 23 & 6.3 & 7.7 & 9.1 \\
\hline 24 & 6.8 & 8.3 & 9.8 \\
\hline 25 & 7.3 & 8.9 & 10.4 \\
\hline 26 & 7.8 & 9.5 & 11.1 \\
\hline 27 & 8.3 & 10.0 & 11.8 \\
\hline 28 & 8.7 & 10.6 & 12.5 \\
\hline 29 & 9.2 & 11.2 & 13.2 \\
\hline 30 & 9.7 & 11.8 & 13.9 \\
\hline 31 & 10.2 & 12.4 & 14.6 \\
\hline 32 & 10.7 & 13.0 & 15.2 \\
\hline 33 & 11.1 & 13.5 & 15.9 \\
\hline 34 & 11.6 & 14.1 & 16.6 \\
\hline 35 & 12.1 & 14.7 & 17.3 \\
\hline 36 & 12.6 & 15.3 & 18.0 \\
\hline 37 & 13.1 & 15.9 & 18.7 \\
\hline 38 & 13.5 & 16.4 & 19.4 \\
\hline 39 & 14 & 17.0 & 20.0 \\
\hline 40 & 14.5 & 17.6 & 20.7 \\
\hline & & & \\
\hline
\end{tabular}

Table 2 Thigh circumference growth curve (Deter et al).(1-3) 
Foetal Thigh Circumference: A Critical Evaluation of Its Relationship to Menstrual Age

\begin{tabular}{|c|r|r|r|r|r|r|}
\hline \multirow{2}{*}{$\begin{array}{c}\text { Menstrual } \\
\begin{array}{c}\text { Age (in } \\
\text { weeks) }\end{array}\end{array}$} & $\begin{array}{l}\text { Lower } \\
\text { Limit }\end{array}$ & $\begin{array}{l}\text { Actual } \\
\text { ThC }\end{array}$ & $\begin{array}{l}\text { Upper } \\
\text { Limit }\end{array}$ & $\begin{array}{l}\text { Standard } \\
\text { deviation }\end{array}$ & $\begin{array}{l}\text { Predicted } \\
\text { Value }\end{array}$ & Difference \\
\hline 22 & 59.43 & 65.18 & 70.93 & 5.75 & 69.66 & 4.48 \\
\hline 23 & 67.09 & 76.35 & 85.61 & 9.26 & 75.51 & -0.84 \\
\hline 24 & 73.75 & 82.29 & 90.83 & 8.54 & 81.37 & -0.92 \\
\hline 25 & 77.36 & 87.81 & 98.26 & 10.45 & 87.23 & -0.58 \\
\hline 26 & 87.53 & 97.06 & 106.59 & 9.53 & 93.09 & -3.97 \\
\hline 27 & 86.20 & 95.37 & 104.54 & 9.17 & 98.95 & 3.58 \\
\hline 28 & 96.08 & 105.13 & 114.18 & 9.05 & 104.80 & -0.33 \\
\hline 29 & 104.87 & 114.79 & 124.71 & 9.92 & 110.66 & -4.13 \\
\hline 30 & 108.40 & 119.00 & 129.60 & 10.60 & 116.52 & -2.48 \\
\hline 31 & 105.57 & 118.00 & 130.43 & 12.43 & 122.38 & 4.38 \\
\hline 32 & 118.65 & 130.26 & 141.87 & 11.61 & 128.24 & -2.02 \\
\hline 33 & 117.02 & 132.71 & 148.40 & 15.69 & 134.09 & 1.38 \\
\hline 34 & 119.79 & 135.84 & 151.89 & 16.05 & 139.95 & 4.11 \\
\hline 35 & 129.21 & 143.38 & 157.55 & 14.17 & 145.81 & 2.43 \\
\hline 36 & 139.58 & 155.55 & 171.52 & 15.97 & 151.67 & -3.88 \\
\hline 37 & 133.74 & 150.89 & 168.04 & 17.15 & 157.53 & 6.64 \\
\hline 38 & 151.95 & 166.00 & 180.05 & 14.05 & 163.38 & -2.62 \\
\hline 39 & 163.10 & 173.90 & 184.70 & 10.80 & 169.24 & -4.66 \\
\hline 40 & 146.70 & 171.00 & 195.30 & 24.30 & 175.10 & 4.10 \\
\hline & & & & & & \\
\hline
\end{tabular}

Table 3 Thigh circumference growth curve (Present study) Based upon Linear=n; $\mathrm{ThC}=-59.22+5.853$ (MA)

\begin{tabular}{|c|r|r|r|r|r|r|}
\hline \multirow{2}{*}{$\begin{array}{c}\text { Menstrual } \\
\begin{array}{c}\text { Age (in } \\
\text { weeks) }\end{array}\end{array}$} & $\begin{array}{l}\text { Lower } \\
\text { Limit }\end{array}$ & $\begin{array}{l}\text { Actual } \\
\text { ThC }\end{array}$ & $\begin{array}{l}\text { Upper } \\
\text { Limit }\end{array}$ & $\begin{array}{l}\text { Standard } \\
\text { deviation }\end{array}$ & $\begin{array}{l}\text { Predicted } \\
\text { Value }\end{array}$ & Difference \\
\hline 22 & 59.43 & 65.18 & 70.93 & 5.75 & 71.25 & 6.07 \\
\hline 23 & 67.09 & 76.35 & 85.61 & 9.26 & 76.29 & -0.06 \\
\hline 24 & 73.75 & 82.29 & 90.83 & 8.54 & 81.44 & -0.85 \\
\hline 25 & 77.36 & 87.81 & 98.26 & 10.45 & 86.71 & -1.10 \\
\hline 26 & 87.53 & 97.06 & 106.59 & 9.53 & 92.10 & -4.96 \\
\hline 27 & 86.20 & 95.37 & 104.54 & 9.17 & 97.60 & 2.23 \\
\hline 28 & 96.08 & 105.13 & 114.18 & 9.05 & 103.21 & -1.92 \\
\hline 29 & 104.87 & 114.79 & 124.71 & 9.92 & 108.92 & -5.87 \\
\hline 30 & 108.40 & 119.00 & 129.60 & 10.60 & 114.75 & -4.25 \\
\hline 31 & 105.57 & 118.00 & 130.43 & 12.43 & 120.68 & 2.68 \\
\hline 32 & 118.65 & 130.26 & 141.87 & 11.61 & 126.71 & -3.55 \\
\hline 33 & 117.02 & 132.71 & 148.40 & 15.69 & 132.84 & 0.13 \\
\hline 34 & 119.79 & 135.84 & 151.89 & 16.05 & 139.08 & 3.24 \\
\hline 35 & 129.21 & 143.38 & 157.55 & 14.17 & 145.41 & 2.03 \\
\hline 36 & 139.58 & 155.55 & 171.52 & 15.97 & 151.85 & -3.70 \\
\hline 37 & 133.74 & 150.89 & 168.04 & 17.15 & 158.38 & 7.49 \\
\hline 38 & 151.95 & 166.00 & 180.05 & 14.05 & 165.00 & -1.00 \\
\hline 39 & 163.10 & 173.90 & 184.70 & 10.80 & 171.72 & -2.18 \\
\hline 40 & 146.70 & 171.00 & 195.30 & 24.30 & 178.53 & 7.53 \\
\hline
\end{tabular}

Table 4 Thigh circumference growth curve (Present study) Based upon Power=n; ThC $=0.61695(\mathrm{MA})^{1.536433}$

Data obtained on measurement errors indicated that the ThC can be measured reliably when it can be measured. The error due to the measurement procedure (based on 40 pairs of measurements) was $0.01 \pm 1.8 \%$. With a conventional dynamic image scanner, however, ThC measurements cannot be made before 16 weeks (HA) and are difficult before 20 weeks. Examination of the raw data revealed a marked difference in the 
relationship of the ThC to menstrual age before and after 22 weeks, the change in ThC with time being significantly greater after 22 weeks than before. Although this could be a true characteristic of the ThC growth curve, the alternative explanation is an inability to obtain ThC measurements on smaller foetuses before 22 weeks. To avoid possible bias in our results, the growth curve studies were limited to the data between 22 and 40 weeks (287 measurements).

Despite an apparent short plateau at term, polynomial regression analysis indicated that adding curvilinear terms did not improve the fit.

\begin{tabular}{|c|c|c|c|c|}
\hline \multirow{2}{*}{$\begin{array}{c}\text { Menstrual } \\
\text { Age (in } \\
\text { weeks) }\end{array}$} & \multicolumn{5}{|c|}{$\begin{array}{c}\text { Predicted Values (mm) } \\
\text { et al. }\end{array}$} & $\begin{array}{c}\text { Vintzileos } \\
\text { et al. }\end{array}$ & $\begin{array}{c}\text { Deter et } \\
\text { al }\end{array}$ & $\begin{array}{l}\text { Present } \\
\text { study }\end{array}$ \\
\hline 20 & 51 & 64 & - & - \\
\hline 21 & 56 & 69 & - & - \\
\hline 22 & 62 & 74 & 71 & 69.66 \\
\hline 23 & 69 & 78 & 77 & 75.51 \\
\hline 24 & 75 & 83 & 83 & 81.37 \\
\hline 25 & 82 & 87 & 89 & 87.23 \\
\hline 26 & 88 & 92 & 95 & 93.09 \\
\hline 27 & 94 & 97 & 100 & 98.95 \\
\hline 28 & 101 & 101 & 106 & 104.8 \\
\hline 29 & 107 & 106 & 112 & 110.66 \\
\hline 30 & 113 & 110 & 118 & 116.52 \\
\hline 31 & 119 & 115 & 124 & 122.38 \\
\hline 32 & 124 & 120 & 130 & 128.24 \\
\hline 33 & 129 & 124 & 135 & 134.09 \\
\hline 34 & 133 & 129 & 141 & 139.95 \\
\hline 35 & 137 & 133 & 147 & 145.81 \\
\hline 36 & 140 & 138 & 153 & 151.67 \\
\hline 37 & 143 & 143 & 159 & 157.53 \\
\hline 38 & 145 & 147 & 164 & 163.68 \\
\hline 39 & 146 & 152 & 170 & 169.24 \\
\hline 40 & 146 & 156 & 176 & 175.10 \\
\hline & & & & \\
\hline
\end{tabular}

Table 5 Predicted values for different thigh circumference growth curves $(2,4,5)$

Coefficient of detramination $\left(\mathrm{R}^{2}\right)$ values for the linear and power functions were 0.987 and 0.984 relatively. The" linear coefficients in all of these models were significantly different from zero, but the curvilinear coefficients were not. The present linear and power function models gave somewhat better fits than the growth model of Rossavik and $\operatorname{Deter}(1,6)$ (R values of 88.9 and $88.8 \%$, respectively), but the $\mathrm{c}$ and $\mathrm{S}$ coefficients of the latter function were not significantly different from 1 and 0 , respectively. In fitting these latter two functions, a starting point for ThC growth of 8.2 weeks (MA) was used, derived from a linear function fitted to the data between 22 and 30 weeks. Based on these results, the optimal models for the ThC growth curve were the following:

(A) In terms of MA

Linear Function:

$$
\begin{aligned}
& \mathrm{I}^{\text {st }} \text { order }=\mathrm{n}: \text { at } 95 \% \text { confidence level } \\
& \quad \mathrm{MA}=10.49+0.1675(\mathrm{ThC}) \\
& \mathrm{II}^{\mathrm{nd}} \text { order } / \text { degree }=\mathrm{n}: \\
& \quad \mathrm{MA}=9.6302+0.1829(\mathrm{ThC})-6.4493 \times 10^{-5}(\mathrm{ThC})^{2} \\
& \mathrm{III}^{\mathrm{rd}} \text { order } / \text { degree }=\mathrm{n}: \\
& \quad \mathrm{MA}=25.9157-0.2648(\mathrm{ThC})+3.8359 \times 10^{-3}(\mathrm{ThC})^{2}-1.0827 \times 10^{-5}(\mathrm{ThC})^{3}
\end{aligned}
$$

Power function:

$$
\mathrm{MA}=1.4851(\mathrm{ThC})^{0.6335}
$$

(B) In terms of $\mathrm{ThC}$

Linear Function:

$$
\mathrm{ThC}=-59.22+5.853 \text { (MA) }
$$


And R2 $=0.987$

Power Function:

$\mathrm{ThC}=0.61695(\mathrm{MA})^{1 \cdot 53643}$

And R2 $=0.984$

The deviation plots, however, indicated that only with the linear function were the deviations symmetrically distributed around zero. An increase in variability with menstrual age was noted that could be eliminated by appropriate predicted values, as has been with other foetal parameters. The mean of the present study was not significantly different from zero. The symmetry of the distribution justifies the use of the standard deviation as the ThC variability parameter. Equation 1 and the standard deviation of the Percent deviation distribution were used to calculate specific normal ranges at different time points between 22 and 40 weeks given in table 3 and 4

\section{Discussion}

Measurement of the ThC provides a potentially straightforward means for assessing the deposition of muscle and fat in the growing foetus. This parameter is preferred over diameter measurements because it is less sensitive to shape changes. A standard plane for measuring the ThC can be defined ultrasonically, and the boundary of the thigh profile obtained is well seen throughout pregnancy, even when the leg is against other soft tissue structures or the fluid volume is decreased. Anatomical studies indicate that the plane of measurement is located at the boundary between the upper and middle thirds of the thigh. Exact positioning of the plane along the long axis of the thigh is not too critical as differences in circumference measurements are not large in the 1-2 cm interval below the level of the standard plane.

Obliquity of the plane with respect to the long axis of the thigh can be minimized by making the femur profile as oval or round as possible and by requiring Chat the profile edges be as sharply defined as possible. Measurement errors have been found to be less than $5^{*}$, indicating that measurement of the $\mathrm{ThC}$ is comparable with measurement of head and abdominal circumferences. Use of ThC is hampered only by the fact that the usual thigh profile is poorly approximated by an ellipse or circle, making calculation of the circumference from diameters highly questionable.

The growth curve for the ThC over the 22-40 weeks (MA) interval can be described by a relatively simple function, although the proportion of variability accounted for by the regression is somewhat lower than that found for other foetal parameters. This probably reflects the greater variability that one would expect in a soft tissue parameter that could be affected by a number of different variables. Restriction to the period after 22 weeks is yet a major problem since abnormalities involving muscle and fat deposition would be expected to occur primarily in the last six weeks of pregnancy when this process is most marked.

ThC growth curves have been presented three previous investigators, but the methods employed by the first two suffered in important ways from those used in the study presented here. Jeanty et al(5) measured the ThC on a profile located at the midlevel of the femur (the means for identifying the location used were not given). The lengths of the long axis of the profile and the axis perpendicular to that axis were determined and the ThC calculated assuming either circular or elliptical profile geometry. The appropriateness of these geometrical assumptions was not evaluated, and the measurements obtained were not compared with direct ThC measurements. Vintzileos et al(4) measured the ThC in the same plane used in the present investigation, but the ThC was either calculated from diameters (assuming circular profile geometry) or measured directly using a map measure. The relative proportions of the two types of measurements were not given, and the validity of assuming circular profile geometry was not evaluated. Deter et al have however done a study upon which the present work is based.

Despite these methodological differences, the growth curves obtained by Jeanty et al (5)and vintzileos et al (4)were similar to that found in the present study. The ThC appeared to be linearly related to menstrual age in all four studies. The differences in predicted values can be explained on the basis of methodological differences (particularly the use of Profile shape assumptions in late pregnancy when crowding and decreases in fluid cause significant distortions in profile shape). The normal range $( \pm 2 \mathrm{D})$ reported in the three previous studies was $1.71 \mathrm{~cm}$ (Jeanty et all, $2.5 \mathrm{~cm}$ (Vintzileos et al) (4, 5)and 1.3-3.1 cm (Deter et al)(1). Differences in measurement procedures and variability assessment can easily account for such differences in the normal range.

What remains to be established is the adequacy of the cross-sectional approach in determining growth curve characteristics (this approach is not always satisfactory as seen with the HC-AC ratio) and the sensitivity of ThC measurements in detecting abnormalities of soft tissue. Comparison of data from cross-sectional and longitudinal studies can provide information on the former, whereas evaluation of the ThC in foetuses at risk for IUGR will allow assessment of the latter. Studies in both areas are currently in progress. 


\section{Conclusion}

The data obtained on measurement errors indicated that the ThC can be measured reliably when it can be measured. Examination of the raw data revealed a mark difference in the relationship of the $\mathrm{ThC}$ to menstrual age before and after 22 weeks, the change in $\mathrm{ThC}$ with time being significantly greater after 22 weeks than before. Although this could be a true characteristic of the ThC growth curve, the alternative explanation is an inability to obtain ThC measurements on smaller foetuses before 22 weeks. To avoid possible bias in our results, the growth curve studies were limited to the data between 22 and 40 weeks (287 measurements).

An increase in variability with menstrual age was noted that could be eliminated by expressing the deviations as percent of the appropriate predicted values, as has been with other foetal parameters. The study concludes that the growth curve for the ThC over the 22-40-week (MA) interval can be described by a relatively simple function, although the proportion of variability accounted for by the regression is somewhat lower than that found for other foetal parameters. what remains to be established is the adequacy of the crosssectional approach in determining growth curve characteristics and the sensitivity of ThC measurements in detecting abnormalities of soft tissues.

\section{Declarations}

a. Funding: Nil

b. Conflict of interest: Nil

c. Ethical approval: Approved

\section{References}

[1]. Deter et al. Fetal Thigh Circumference : A critical evaluation of its relationship to menstrual age. J Clin U ltrasound. 1986;14:10510 .

[2]. Deter et al. evaluation of thigh circumference measurements : a comparative ultrasound and anatomical study. J Clin U ltrasound. 1986;14:99-.

[3]. Deter et al. Longitudinal studies of thigh cirumference growth in normal foetuses. J Clin U ltrasound. 1987;15:388-93.

[4]. Vintzileos AM et al. Ultrasound foetal thigh -calf circumferences and gestational age-independent fetal ratios in normal pregnancy. J Ultrasound Med. 1985(4).

[5]. Jeanty P et al. Fetal limb volume : a new parameter to assess fetal growth and nutrition. J Ultrasound Med. 1985;4:273.

[6]. Rossvik JK et al. Mathematical modelling of fetal growth. III. Evaluation of head growth using head profile area. J Clin U ltrasound. 1987(15):5-12. 